\title{
PHARMACOKINETIC PROFILE OF METFORMIN HYDROCHLORIDE IN DRIED BLOOD SPOT OF HEALTHY SUBJECTS USING HIGH-PERFORMANCE LIQUID CHROMATOGRAPHY-PHOTODIODE ARRAY
}

\author{
YAHDIANA HARAHAP*, CITRA DARA MALIA, SUNARSIH \\ Bioavailability/Bioequivalence Laboratory, Faculty of Pharmacy, Universitas Indonesia, Depok 16424, Indonesia. \\ Email: yahdiana03@yahoo.com
}

Received: 08 June 2018, Revised and Accepted: 09 August 2018

ABSTRACT

Objective: This study aimed to analyze the metformin hydrochloride in dried blood spot (DBS) sample and evaluate a pharmacokinetic profile in six healthy subjects who administered 850-mg metformin hydrochloride in tablet using high-performance liquid chromatography-photodiode array (HPLC-PDA).

Methods: Metformin analysis was performed on data of six healthy subjects who administered $850 \mathrm{mg}$ of metformin hydrochloride as tablets. Blood samples were taken at 12-time points at intervals of up to $12 \mathrm{~h}$, and data were analyzed using HPLC-PDA.

Results: A linear calibration curve was obtained in the range of $25-5000 \mathrm{ng} / \mathrm{mL}$, with $\mathrm{r}=0.9990$. Pharmacokinetic profiles of metformin were obtained

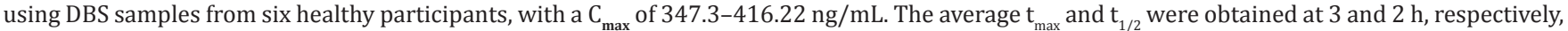
and the area under the curve (AUC) $)_{0-t} / \mathrm{AUC}_{0-\infty}$ ratio was $>80 \%$ for all subjects.

Conclusion: The DBS biosampling technique can be developed for application in pharmacokinetic study of metformin $\mathrm{HCl}$.

Keywords: Dried blood spot, High-performance liquid chromatography, Metformin hydrochloride, Pharmacokinetic profile, Validation.

(c) 2018 The Authors. Published by Innovare Academic Sciences Pvt Ltd. This is an open access article under the CC BY license (http://creativecommons. org/licenses/by/4. 0/) DOI: http://dx.doi.org/10.22159/ijap.2018.v10s1.78

\section{INTRODUCTION}

Diabetes mellitus (DM) is a disorder of carbohydrate, protein, and fat metabolism due to decreased insulin secretion, insulin receptor sensitivity, or both and can be identified by hyperglycemia in the patient [1]. In 2015, the International Diabetes Federation rated Indonesia as the country with the seventh greatest number of people with diabetes aged 20-79 years, with 10 million people with the disease. By 2040, the number of people with diabetes in Indonesia is projected rise to 16.3 million. Furthermore, $90-95 \%$ of all cases of diabetes are type 2 DM [2].

Metformin is the first-line medication for patients with type $2 \mathrm{DM}$ and is the drug of choice for type 2 DM patients with obesity [3]. Metformin is included in the National List of Essential Medicines, the list of drugs that are assessed using a bioequivalence (BE) test, and was one of the most commonly prescribed drugs worldwide in 2014 [4-8].

Pharmacokinetic studies comprise a series of tests to determine the pharmacokinetic parameters of a drug [4]. They generally use plasma samples, and biosampling techniques are performed by taking a volume of approximately 5-15 $\mathrm{mL}$ of blood through venipuncture, often causing discomfort for the subject. However, over the past 5-7 years, a dried blood spot (DBS) technique has been intensively developed and evaluated for the quantification of drug exposure in non-clinical as well as clinical studies at various phases for drug research and development. DBS is an innovative biosampling technique in which small quantities $(10-80 \mu \mathrm{L})$ of wet blood samples are collected on DBS paper or other paper materials then dried for 2-3 h at room temperature or under nitrogen gas flow with controlled air humidity [9]. This method is preferable as it is minimally invasive for the patient (using a sterile lancet), the volume of blood required is low, it is simple and does not cost much to implement, and the analytes in the dry blood sample are relatively stable in the long term. Metformin is reported to be partitioned into erythrocytes; therefore, the use of DBS could be considered instead of plasma samples to measure metformin concentrations $[10,11]$.

Metformin hydrochloride analysis using DBS samples requires a device that has high selectivity and sensitivity due to the low blood volume and the fact it is derived from the periphery [12]. High-performance liquid chromatography (HPLC) is the most widely used technique for drug analysis in biological matrices. It is a commonly used instrument that is available in the laboratory and analytical methods are more applicable [13]. Photodiode array (PDA) detectors are commonly used and are advantageous in sensitive and selective HPLC systems for the analysis of drugs and their metabolites in biological matrices [14].

To date, there have been no reports of pharmacokinetic tests of metformin hydrochloride using the DBS biosampling technique. Further, analysis is required to determine whether metformin hydrochloride bioanalysis using the DBS biosampling technique can be applied in vivo as well as more widely.

In the present study, six healthy subjects administered $850 \mathrm{mg}$ of metformin hydrochloride in tablet formulation. Blood samples were taken at 12 -time points at various intervals up to $12 \mathrm{~h}$. The study was approved Ethical Clearance from the Medical Research Ethics Committee of the Faculty of Medicine, Universitas Indonesia (no. 63/UN2.FI/ETIK/2017).

The aim of the present study was to the analyze metformin hydrochloride in DBS sample and evaluate a pharmacokinetic profile in six healthy subjects who administered 850-mg metformin hydrochloride in tablet using HPLC-PDA.

\section{METHODS}

\section{Instruments}

HPLC (Waters 2695 Separation Mode) consisting of pumps, an autosampler, C18 column (Waters, SunfireTM $5 \mu \mathrm{m} ; 250 \times 4.6 \mathrm{~mm}$ ), 
PDA (Waters 2996), and a computer for data processing. Other equipment used included a pH meter (Eutech $\mathrm{pH}$ 510), evaporator (Turbovap Caliper), analytical scales (Acculab), eluent filters and samples (Whatman), degasser (Elmasonic S60H), vortex (Maxi Mix II), Eppendorf micropipettes (Socorex), microtubes, sample cups, pipette tips, and glassware.

\section{Materials}

\section{Test materials}

DBS samples were obtained from blood samples collected on DBS paper from six healthy subjects who administered $850 \mathrm{mg}$ of metformin hydrochloride in tablet.

\section{Chemical materials}

The materials used included metformin hydrochloride and calcium atorvastatin were purchased from British Pharmacopoeia, HPLC grade acetonitrile (Merck), HPLC grade methanol (Merck), sodium dihydrogen phosphate monohydrate (Merck), sodium dodecyl sulfate (SDS) (SDS; Merck), orthophosphoric acid (Merck), sodium hydroxide $(\mathrm{NaOH})$ (NaOH; Merck), aquabidest (Ikapharmindo), DBS paper (Perkin Elmer), human blood (Indonesian Red Cross), and 850-mg immediate release metformin hydrochloride tablets (Merck).

\section{Sampling materials}

Sampling materials included sterile pens and lancets (sterile needle; Medilance), Eppendorf micropipettes (Socorex), alcohol swabs (OneMed), and 0.5-mL vacutainers (GM Micro Tube).

\section{Stock solution of metformin $\mathrm{HCl}$ and atorvastatin}

10 milligrams of standard metformin hydrochloride were carefully weighed and added into a $10-\mathrm{mL}$ measuring flask. The substance was dissolved in aquabidest, to a final volume of $10 \mathrm{~mL}$, giving a standard compound solution of $1000 \mu \mathrm{g} / \mathrm{mL}$. The stock solution was diluted to obtain solutions of various concentrations for a calibration curve. $10 \mathrm{mg}$ of standard atorvastatin compound were also carefully weighed and dissolved with methanol to a final volume of $10 \mathrm{~mL}$. The resulting stock solution of $1000 \mu \mathrm{g} / \mathrm{mL}$ was diluted to obtain solutions of various concentrations.

\section{Chromatography conditions}

HPLC-PDA was performed using a detector with a wavelength of $234 \mathrm{~nm}$. The column used was a C18 (Waters, SunfireTM $5 \mu \mathrm{m}$; $250 \times 4.6 \mathrm{~mm}$ ). The mobile phase was $10-\mathrm{mM}$ SDS and $10-\mathrm{mM}$ phosphate buffer in water-acetonitrile $(60: 40)$ and then was adjusted to $\mathrm{pH} 7.0$ with orthophosphoric acid and $\mathrm{NaOH}$; the column temperature was $40^{\circ} \mathrm{C}$; flow rate was $0.8 \mathrm{~mL} / \mathrm{min}$, with an analysis time of $10 \mathrm{~min}$.

\section{Sample preparation}

A volume of $20 \mu \mathrm{L}$ of blood containing analytes was pipetted and spotted on DBS paper. The samples were then dried at room temperature $\left(20-25^{\circ} \mathrm{C}\right)$ for $3 \mathrm{~h}$. After drying, the paper was cut according to the marking on the paper, then inserted into a microtube, and $50 \mu \mathrm{L}$ of atorvastatin $(10 \mu \mathrm{g} / \mathrm{mL})$ as an internal standard and $0.5 \mathrm{~mL}$ of methanol $(60 \%)$ as the extracting solvent were added. Sonication was performed for $10 \mathrm{~min}$. The process was continued by mixing the solution using a vortex for $90 \mathrm{~s}$. The solution was then transferred into the microtubes and evaporated to dryness at $60^{\circ} \mathrm{C}$ for $15 \mathrm{~min}$ under nitrogen gas. The residue was reconstituted by adding $200 \mu \mathrm{L}$ of mobile phase followed by sonication for $3 \mathrm{~min}$. The solution was then transferred to an autosampler vial and centrifuged for $10 \mathrm{~min}$ (room temperature, $3000 \mathrm{rpm}, 10 \mathrm{~min}$ ). A 100- $\mu \mathrm{L}$ sample volume was injected into HPLC system.

\section{Partial validation of bioanalysis methods}

\section{Calibration curve and linearity test}

Blank samples (whole blood without analyte), zero samples (whole blood with internal standard), and metformin hydrochloride solution in whole blood at concentrations of $25,50,75,100,500,1000,2500,3750$, and $5000 \mathrm{ng} / \mathrm{mL}$ were collected. A calibration curve was obtained by spiking each blood sample to obtain the desired concentration. Then, $20 \mu \mathrm{L}$ of the spiked blood was collected on DBS paper and dried for around $3 \mathrm{~h}$. The paper was cut according to the marking on the paper and inserted into microtubes. Next, $50 \mu \mathrm{L}$ of atorvastatin $(10 \mu \mathrm{g} / \mathrm{mL})$ was added as an internal standard and extracted using the same extraction method as used for the sample preparation. A 100- $\mu \mathrm{L}$ sample volume was injected into the HPLC system.

The regression value of the peak area (y) to the concentration of analyte in plasma $(\mathrm{x})$ was calculated for each concentration, and a calibration curve was prepared. A linearity test was performed using data from the calibration curve, then analyzed using peak area regression to the metformin concentration in the DBS sample, and a correlation coefficient (r) was obtained, demonstrating its linearity. In addition, calculation of the percentage difference (\% diff) with the limit value of $\%$ diff did not exceed $20 \%$ for the lower limit of quantification (LLOQ) and $15 \%$ for all other concentrations.

\section{Within-run accuracy and precision}

Metformin hydrochloride solutions in whole blood were made with LLOQ concentration and metformin quality control (QC) solutions with low, medium, and high $(\mathrm{QC}$ low $[\mathrm{QCL}], \mathrm{QC}$ medium [QCM], and QC high [QCH], respectively) concentrations. A volume of $20 \mu \mathrm{L}$ of blood was spotted on DBS paper, dried for around $3 \mathrm{~h}$, and the paper was cut according to the marking on the paper. The paper was inserted into microtubes, then $50 \mu \mathrm{L}$ of atorvastatin $(1 \mu \mathrm{g} / \mathrm{mL})$ was added as an internal standard and extracted using the same method as the sample preparation. Next, a 100- $\mu \mathrm{L}$ sample volume was injected into HPLC system. Accuracy and precision tests were performed using five replicas at each concentration in one run (intrabatch/within-run accuracy). The $\%$ diff of data obtained was calculated to determine the accuracy. The precision was measured using the calculation of the coefficient of variation $(\% \mathrm{CV})$ data obtained. The requirement $\%$ diff and $\% \mathrm{CV}$ values were $<20 \%$ for LLOQ and $<15 \%$ for all other concentrations.

\section{Treatment of healthy subjects}

The scope of the study was explained to the six healthy subjects, and all gave signed informed consent and passed the screening. The day before the sampling, the subjects quarantined at the Bioavailability and Bioequivalence Laboratory of the Faculty of Pharmacy, Universitas Indonesia. After an 8-h fast, each subject received an oral dose of 850 -mg metformin hydrochloride tablets, with $240 \mathrm{~mL}$ of water. To prevent hypoglycemia, subjects were given $100 \mathrm{~mL}$ of a $10 \%$ glucose solution every hour for $3 \mathrm{~h}$. Subsequently, at 4,8 , and $12 \mathrm{~h}$ after administration of the 850-mg metformin hydrochloride tablet, subjects were fed standard foods. Standardization of body position and physical activity throughout the study day was ensured due to their effects on motility and blood flow of the gastrointestinal tract.

\section{Sampling and preparation of DBS samples from healthy subjects} Blood samples were taken at 0 (predose), 0.5, 1, 1.5, 2, 2.5, 3, 3.5, 4, 6, 8 , and $12 \mathrm{~h}$ after administration of 850-mg metformin hydrochloride tablets in six healthy subjects (12-point collection). Blood collection was performed through finger prick using a sterile lancet needle. The amount of blood used for this study was approximately $40 \mu \mathrm{L}$. Blood was collected in $0.5-\mathrm{mL}$ vacutainers, and $20 \mu \mathrm{L}$ was spotted on DBS paper and dried for approximately $3 \mathrm{~h}$. The paper was cut out according to the marking on the paper, inserted into microtubes.

\section{Metformin hydrochloride analysis in the sample}

Analysis was performed using HPLC-PDA $(100-\mu \mathrm{L}$ sample injection volume) with an optimized mobile phase to determine the concentration of metformin hydrochloride in healthy subjects who administered 850mg metformin hydrochloride in tablet to evaluate the pharmacokinetic profile. From the peak area obtained, the concentration of metformin hydrochloride was calculated using a standard calibration curve. 
Calculation of pharmacokinetic parameters

Metformin hydrochloride blood concentration data were plotted to form a pharmacokinetic profile curve (concentration vs. time). From these data, the pharmacokinetic parameters of the maximum concentration in blood $\left(\mathrm{C}_{\max }\right)$ and the maximum time $\left(\mathrm{t}_{\max }\right)$ were calculated. Other pharmacokinetic parameters determined included half-life $\left(\mathrm{t}_{1 / 2}\right)$, area under the curve $(\mathrm{AUC})_{0-\mathrm{r}^{\prime}}$ and $\mathrm{AUC}_{0-\infty}$.

\section{RESULTS AND DISCUSSION}

\section{Calibration curves}

The results indicated that the range of the calibration curve for metformin was 25-5000 ng/mL. The calibration curve consisted of blanks (whole blood without analyte and internal standard), zero samples (whole blood with internal standard), and non-zero blood (whole blood with analyte and internal standard) using nine different concentrations: 25 , $50,75,100,500,1000,2500,3750$, and $5000 \mathrm{ng} / \mathrm{mL}$. The calibration curve yielded a linear regression equation of $y=0.0003 x+0.0114$ with $r=0.9990$, where $\mathrm{x}$ represents the concentration of metformin $(\mathrm{ng} / \mathrm{mL})$ and y represents the peak area ratio between metformin and atorvastatin. These data show that the calibration curve obtained met the requirements of $\leq 20 \%$ at concentrations of LLOQ and $\leq 15 \%$ at other concentrations.

\section{Within-run accuracy and precision}

In the present study, only partial validation was performed, including precision and intraday accuracy. According to the 2011 guideline of Bioanalytical Method Validation from the EMEA, partial validation can be performed, starting with intraday accuracy and precision parameters until almost all validation parameters are full. Full validation was not performed because the analytical methods were fully validated in other studies using the same research conditions including location, instruments, methods of extraction, and bioanalysis methods. An accuracy test was performed to assess the similarity between the measured and actual concentrations by determining its bias value ( $\%$ diff). A precision test was conducted to determine the proximity of the repetition of the measurement analytes by analyzing the value of $\%$ $\mathrm{CV}$. The accuracy and precision tests were performed using five replicas of four different concentrations of metformin hydrochloride in whole blood, namely LLOQ QCL, QCM, and QCH. It was essential that both accuracy and precision met the requirement stated in the guidelines by the EMEA, that is, $\leq 20 \%$ at concentrations of LLOQ and $\leq 15 \%$ at other concentrations.

The results of the within-run accuracy testing on metformin met the requirements; the \% diff value at LLOQ concentration was $12.35 \%-$ $17.43 \%$, and the QC concentration was $1.48-13.60 \%$. The within-run precision test also met the requirements for the analyte with a $\% \mathrm{CV}$ value for metformin at $2.79 \%$ for LLOQ $2.75 \%$ for QCL, $1.56 \%$ for QCM, and $2.37 \%$ for QCH. Although the obtained \% CV and \% diff were different from previous studies, the accuracy and precision test results in the analytes remained within the required range, and still qualified, demonstrating that the method of analysis was accurate and precise.

\section{Analysis of samples from healthy subjects}

Sampling process

Blood samples were taken under the supervision of a doctor. The subjects then received an oral dose of 850-mg metformin hydrochloride in tablet (Glucophage) with $240 \mathrm{~mL}$ of water. To prevent hypoglycemia, subjects were given $100 \mathrm{~mL}$ of a $10 \%$ glucose solution every hour for $3 \mathrm{~h}$. Subsequently, at 4, 8, and $12 \mathrm{~h}$ after administration of metformin hydrochloride, the subjects were fed standard meals. Body position and physical activity were standardized throughout the study day due to their effects on motility and blood flow of the gastrointestinal tract.

Up to $40 \mu \mathrm{L}$ of blood was collected at 12-time points: 0 (predose), 0.5, $1,1.5,2,2.5,3,3.5,4,6,8$, and $12 \mathrm{~h}$ after administration of 850-mg metformin hydrochloride tablets. Blood collection was performed through finger prick using a sterile lancet needle. The collected blood was then transferred to $0.5-\mathrm{mL}$ vacutainers. Side effects were monitored during the study based on direct questions, spontaneous reports, and clinical parameters measured during the sampling process. Adverse event during the sampling process or complaints from the subject were monitored and studied by the doctor.

After blood was collected, $20 \mu \mathrm{L}$ was directly spotted on DBS paper and dried in a horizontal position for $3 \mathrm{~h}$ in an open space at room temperature $\left(20-25^{\circ} \mathrm{C}\right)$. Samples were stored in an airtight plastic (sealable plastic) with a silica pack to control the moisture during storage. Humidity was well controlled to reduce the risk of analytical degradation due to bacterial growth that may have affected the results of the analysis. The dried blood samples were stored at room temperature in dry and cool $\left(20-25^{\circ} \mathrm{C}\right)$ conditions before analysis. The DBS sample was then prepared for partial validation methods.

\section{Sample analysis results}

Analysis was performed on one batch to generate three calibration curves (CC1, CC2, and CC3). Sample QC 1 consisted of QCL, QCM, and $\mathrm{QCH}$ of each of two replicas, and 12 sample points of subject 1; sample QC 2 consisted of QCL, QCM, and QCH of each of two replicas, 12 sample points of participant 2; and sample QC 3 consisted of QCM and QCH of each of the two replicas. The calibration curve yielded a linear regression equation of $y=0.0003 x+0.0116$, with $r=0.9980$ for CC1; $y=0.0003 x+0.0119$, with $r=0.9990$ for CC2; and $y=0.0003 x+0.0112$, with $r=0.9992$ for CC3. Sample analysis was completed within 1 month after sampling. The analysis time still met the requirements as the longterm stability validation was 45 days.

Fig. 1 shows the metformin hydrochloride concentrations in blood plotted on a pharmacokinetic profile curve (concentration vs. time). The data show the pharmacokinetic parameters, that is, AUC, the maximum concentration in blood $\left(\mathrm{C}_{\max }\right)$, maximum time $\left(\mathrm{t}_{\max }\right)$, and halftime $\left(\mathrm{t}_{1 / 2}\right)$. The calculated pharmacokinetic parameters for each participant are shown in Table 1.

The results showed that the $\mathrm{C}_{\max }$ obtained from six healthy subjects ranged from 347.3 to $416.22 \mathrm{ng} / \mathrm{mL}$. The mean $\mathrm{C}_{\max }$ was $367 \mathrm{ng} / \mathrm{mL}$ with a standard deviation of $26.14 \mathrm{ng} / \mathrm{mL}$. The lowest $\mathrm{C}_{\max }$ concentration was $347.3 \mathrm{ng} / \mathrm{mL}$, observed in subject CF1, and the highest concentration was $416.22 \mathrm{ng} / \mathrm{mL}$, observed in subject CF4. There was a slight difference in the maximum concentration measured in each participant. This was due to variations in the body's response to metformin and metabolic processes occurring in each participant, resulting in variations in concentrations. The $t_{\text {max }}$ in the six test subjects was observed at the sixth and seventh blood sampling time points, that is, at $2.5 \mathrm{~h}$ after drug administration for subjects CF1, CF2, CF4, and CF5 and $3 \mathrm{~h}$ after drug administration for subjects CF3 and CF6. The mean $t_{\text {max }}$ for the six subjects was $3 \mathrm{~h}$. This was in accordance with the literature showing that $\mathrm{t}_{\max }$ was obtained around 2-3 $\mathrm{h}$ after drug

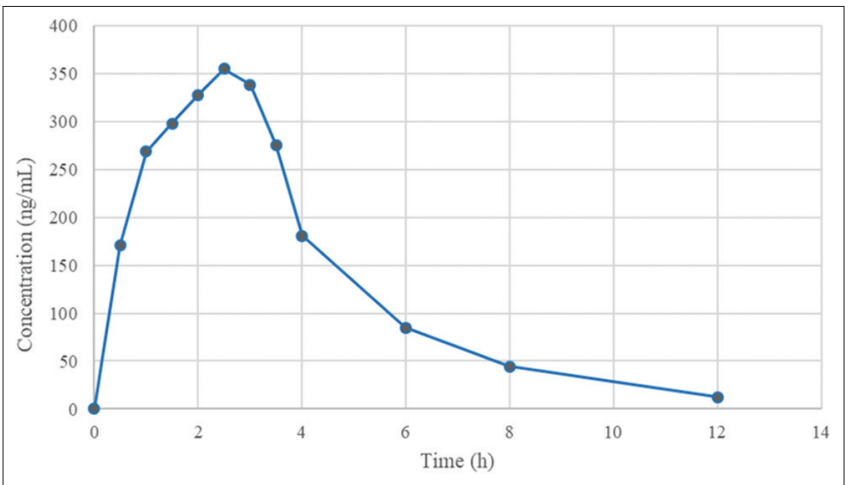

Fig. 1: Mean pharmacokinetic profile versus time of metformin HCL on six healthy subjects in dried blood spot samples 
Table 1: Results of pharmacokinetic parameters

\begin{tabular}{|c|c|c|c|c|c|c|}
\hline Subject & $\mathrm{C}_{\max }(\mathrm{ng} / \mathrm{mL})$ & $\mathbf{t}_{\max }(\mathbf{h})$ & $\mathbf{t}_{1 / 2}(\mathrm{~h})$ & $\mathrm{AUC}_{0-\mathrm{t}}(\mathrm{ng} \cdot \mathrm{h} / \mathrm{mL})$ & $\operatorname{AUC}_{0-\infty}(\mathrm{ng} \cdot \mathrm{h} / \mathrm{mL})$ & $\mathrm{AUC}_{0-\mathrm{t}} / \mathrm{AUC}_{0-\infty}(\%)$ \\
\hline CF1 & 347.3 & 2.5 & 1.67 & 1309.36 & 1309.36 & 100 \\
\hline $\mathrm{CF} 2$ & 348.4 & 2.5 & 1.67 & 1349.87 & 1349.87 & 100 \\
\hline CF3 & 362.94 & 3 & 2.80 & 1729.3 & 1833.51 & 94.32 \\
\hline CF4 & 416.22 & 2.5 & 2.94 & 1861.79 & 1968.31 & 94.59 \\
\hline CF5 & 352.64 & 2.5 & 1.65 & 1404.53 & 1404.53 & 100 \\
\hline CF6 & 373.02 & 3 & 2.48 & 1772.04 & 1861.68 & 95.18 \\
\hline Mean \pm SD & $367 \pm 26.14$ & $3 \pm 0.26$ & $2 \pm 0.61$ & $1571 \pm 242.94$ & $1621 \pm 297.05$ & $97.35 \pm 2.92$ \\
\hline
\end{tabular}

$\%$ CV: Coefficient of variation, SD: Standard deviation, AUC: Area under the curve

administration [15]. Our results showed that the $\mathrm{AUC}_{0-\mathrm{t}} / \mathrm{AUC}_{0-\infty}$ met the requirements of $>80 \%$ for all test subjects.

The $\mathrm{C}_{\max }$ values obtained from the six subjects showed that the achieved LLOQ was in the range of $6.00-7.10 \%$ of the maximum concentration of the analyte in the body $\left(\mathrm{C}_{\max }\right)$, while the EMEA requirement is $5 \%$. As there were no previous studies on the pharmacokinetic profile of metformin hydrochloride with biosampling of DBS, the concentration of metformin in plasma was used as a reference for $\mathrm{C}_{\max }$. However, we demonstrated that the $\mathrm{C}_{\max }$ for metformin using DBS was lower than the $\mathrm{C}_{\max }$ for metformin in plasma; therefore, a more sensitive LLOQ was required in the range of $17.4-20.91 \mathrm{ng} / \mathrm{mL}$. This could have been caused by unextracted analytes in the DBS paper, making the LLOQ measurements less sensitive. The DBS paper used was polar and made of cellulose, so there was a high probability of metformin having polar properties, strongly bound to the DBS paper.

The metformin concentrations in DBS were lower than those in plasma. Following administration of 850-mg metformin hydrochloride tablets, the $C_{\max }$ of metformin in plasma was $1000-1500 \mathrm{ng} / \mathrm{mL}$ (Porta et al., 2008). A major contributor to the lower concentration of metformin in DBS samples compared with plasma is due to the blood samples being taken from the periphery and in small volumes. Whole blood from the periphery comprises blood cells, plasma, and interstitial fluid, as well as more intrusive components. In addition, there was a difference in analyte concentration in the periphery (capillary vessels) with the veins $[12,16]$. The distribution coefficient $(\mathrm{cP} / \mathrm{cBC})$ for metformin was $4.65 \pm 0.73$. This suggests that the plasma concentration of metformin is 4 times higher than that of the blood cell concentration [11]. This may have contributed to the lower concentration of metformin observed with DBS biosampling compared with venous biosampling. Therefore, a multiplier factor is required to convert the concentration from the DBS analyzer to the plasma analyzer concentration.

The DBS method has more advantages than vein biosampling method. It is a more comfortable method of biosampling as it is minimally invasive, especially during the pharmacokinetic and BE tests, where blood were collected for several points (12-18 times) over a period of time. The technique is also simpler and does not cost much to implement, and the analytes in the sample tend to be more stable compared with other biosampling techniques [11]. Metformin is partitioned in erythrocytes; therefore, DBS biosampling for metformin analysis is possible and can be used for pharmacokinetic, BE, or other metformin clinical trials. This method has been demonstrated to meet the validation criteria and can be applied in vivo. However, future studies are required to develop further methods of extraction to improve the recovery so to achieve LLOQ analysis.

\section{CONCLUSION}

The DBS biosampling technique can be developed for application in pharmacokinetic study of metformin $\mathrm{HCl}$.

\section{CONFLICTS OF INTEREST}

All authors declare that they have no conflicts of interest.

\section{REFERENCES}

1. Dipiro J, Dipiro CV, Wells BG, Scwinghammer TL. Pharmacotherapy a Pathophysiologic Approach. $9^{\text {th }}$ ed. United States: The McGraw-Hill Companies, Inc.; 2015.

2. Muchid A. Pharmaceutical Care for Diabetes Mellitus. Jakarta: Indonesian Ministry of Health; 2005.

3. Aburuz S, Millership J, McElnay J. Dried blood spot liquid chromatography assay for therapeutic drug monitoring of metformin. J Chromatogr B Analyt Technol Biomed Life Sci 2006;832:202-7.

4. Indonesian National Agency of Food and Drug Control. Regulation from Head of No. HK.03.1.23.12.11.10217 about Drug Obligate Equivalence Study Jakarta. Jakarta: Indonesian National Agency of Food and Drug Control; 2011.

5. Tan MH, Alquraini H, Mizokami-Stout K, MacEachern M. Metformin: From research to clinical practice. Endocrinol Metab Clin North Am 2016;45:819-43.

6. Earle RR, Bandaru KK, Lakshmi UA. Formulation and characterization of sustained release coated matric granules of metformin hydrochloride. Asian J Pharm Clin Res 2018;11:387-92.

7. Kotecha NC, Patel JK. Method development and validation of a reversed-phase liquid chromatographic method for the simultaneous estimation of selected anti diabetic drugs in the presence of their degradation products. Asian J Pharm Clin Res 2018;11:345-52.

8. Bookya P, Raparla R, Sriamula HP, Tarrigopula S, Vanga S. Formulation and evaluation of metformin hydrochloride sustained release oral matrix tablets. Asian J Pharm Clin Res 2018;11:342-5.

9. Evans C, Arnold M, Bryan P, Duggan J, James CA, Li W, et al. Implementing dried blood spot sampling for clinical pharmacokinetic determinations: Considerations from the IQ consortium microsampling working group. AAPS J 2015;17:292-300.

10. Center of Drug Evaluation and Research. Metformin Hydrochloride Tablets. United States: Food and Drug Administration; 2002.

11. Scherf-Clavel M, Högger P. Analysis of metformin, sitagliptin and creatinine in human dried blood spots. J Chromatogr B Analyt Technol Biomed Life Sci 2015;997:218-28.

12. Wilhelm AJ, den Burger JC, Swart EL. Therapeutic drug monitoring by dried blood spot: Progress to date and future directions. Clin Pharmacokinet 2014;53:961-73.

13. Alshishani A, Makahleh A, Yap HF, Gubartallah EA, Salhimi SM, Saad B, et al. Ion-pair vortex assisted liquid-liquid microextraction with back extraction coupled with high performance liquid chromatography-UV for the determination of metformin in plasma. Talanta 2016;161:398-404.

14. Evans G. A Handbook of Bioanalysis and Drug Metabolism. United States of America: CRC Press; 2014.

15. Porta V, Schramm S, Kano E, Koono E, Armando Y, Fukuda K, et al. HPLC-UV determination of metformin in human plasma for application in pharmacokinetics and bioequivalence studies. J Pharm Biomed Anal 2008;46:143-7.

16. Sharma A, Jaiswal S, Shukla M, Lal J. Dried blood spots: Concepts, present status, and future perspectives in bioanalysis. Drug Test Anal 2014;6:399-414. 